\title{
A NEW COUNTING CHAMBER FOR THE EOSINOPHIL COUNT
}

\author{
BY
}

T. MANNERS

From the Department of Pathology, Royal Infirmary, Sunderland

(RECEIVED FOR PUBLICATION JULY 20, 1951)

During the past four years numerous articles have been published in which eosinophil counts have been used in the control of therapy by A.C.T.H. In most of these the counts have been performed by using four Fuchs-Rosenthal counting chambers for each count. The author also used these counting chambers for the experimental work on a new diluent for eosinophil counts (Manners, 1951), but found the method cumbersome. Much time was wasted in filling and cleaning four counting chambers when only one should have been necessary. While counting, it was necessary to move to the next counting chamber three times and to refocus if the counting chambers were of different thickness. These disadvantages are obviated by the use of a new counting chamber* designed by the author.

The ruling in the new chamber is similar to that of the Fuchs-Rosenthal in that it is composed of large squares $1 \mathrm{~mm} . \times 1 \mathrm{~mm}$., each divided into 16 smaller squares. The ruled area is $4 \mathrm{~mm} . \times 12.5 \mathrm{~mm}$., and the depth of the chamber is $0.2 \mathrm{~mm}$. The whole ruled area is counted and the volume enclosed by it is $10 \mathrm{c} . \mathrm{mm}$. As the dilution of blood is $1: 10$, no calculation is required to find the number of eosinophils per c.mm. When using four Fuchs-Rosenthal chambers the number counted has to be divided by 1.28 to obtain the number of eosinophils per c.mm.

Plum (1936) showed that the accuracy attainable in chamber counting depends for the most part on the number of cells per sample counted. There is a great variation due to chance distribution in the number of cells from sample to sample of a given blood, and its standard deviation (S.D.) is equal to the square root of the mean number of cells per sample. As the volume of fluid contained in the new counting chamber is slightly smaller than the total volume contained in four FuchsRosenthal counting chambers, the accuracy of the count is slightly less. The theoretical accuracies of the two counting techniques can be compared by taking an example.

With a blood containing 100 eosinophils per c.mm., the counts obtained in the new counting chamber would average 100 cells. The S.D. of their variation due to chance would be $\sqrt{100}=10$, which is $10 \%$ of the mean. Using four Fuchs-Rosenthal chambers, which have a total volume of $12.8 \mathrm{c} . \mathrm{mm}$., the count would average 128 cells. The S.D. in this case would be $\sqrt{128}=11.3$, which is $8.8 \%$ of the mean. With 100 eosinophils per c.mm. the comparison is thus between an S.D. of $10 \%$ and $8.8 \%$.

The difference will be less at higher levels and greater at lower levels. The difference is small and the accuracy attainable in the new chamber is sufficient for routine counting of eosinophils.

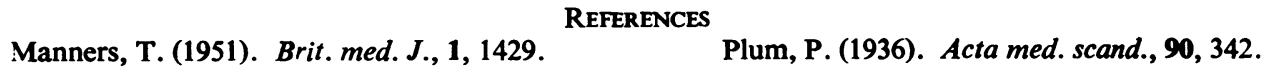

* Made by Messrs. Hawksley and Sons, Ltd., 17, New Cavendish Street, London, W.1. 